\title{
BOUNDARY PARTICLE METHOD FOR INVERSE CAUCHY PROBLEMS OF INHOMOGENEOUS HELMHOLTZ EQUATIONS
}

\section{Wen Chen}

Center for Numerical Simulation Software in Engineering and Sciences, Department of Engineering Mechanics, College of Civil Engineering, Hohai University, Nanjing 210098, P.R. China, chenwen@hhu.edu.cn

\section{Zhuojia Fu}

Center for Numerical Simulation Software in Engineering and Sciences, Department of Engineering Mechanics, College of Civil Engineering, Hohai University, Nanjing 210098, P.R. China

Follow this and additional works at: https://jmstt.ntou.edu.tw/journal

Part of the Engineering Commons

\section{Recommended Citation}

Chen, Wen and Fu, Zhuojia (2009) "BOUNDARY PARTICLE METHOD FOR INVERSE CAUCHY PROBLEMS OF INHOMOGENEOUS HELMHOLTZ EQUATIONS," Journal of Marine Science and Technology. Vol. 17: Iss. 3, Article 1. DOI: $10.51400 / 2709-6998.1952$

Available at: https://jmstt.ntou.edu.tw/journal/vol17/iss3/1

This Research Article is brought to you for free and open access by Journal of Marine Science and Technology. It has been accepted for inclusion in Journal of Marine Science and Technology by an authorized editor of Journal of Marine Science and Technology. 


\title{
BOUNDARY PARTICLE METHOD FOR INVERSE CAUCHY PROBLEMS OF INHOMOGENEOUS HELMHOLTZ EQUATIONS
}

\author{
Wen Chen* and Zhuojia Fu*
}

\begin{abstract}
Key words: boundary particle method, truncated singular value decomposition, inhomogeneous Helmholtz equations, inverse problem.
\end{abstract}

\begin{abstract}
This paper investigates the boundary particle method (BPM) coupled with truncated singular value decomposition (TSVD) regularization technique on the solution of inverse Cauchy problems of inhomogeneous Helmholtz equations. Unlike the other boundary discretization methods, the BPM does not require any inner nodes to evaluate the particular solution, since the method uses the recursive composite multiple reciprocity technique to reduce an inhomogeneous problem to a series of higher-order homogeneous problems. The BPM is particular attractive to solve inverse problems thanks to its truly boundary-only meshless merit. In this study, numerical experiments demonstrate that the BPM in conjunction with the TSVD is highly accurate, computationally efficient and stable for inverse Cauchy problems.
\end{abstract}

\section{INTRODUCTION}

Inverse problems, especially Cauchy problems, are typical ill-posed problems and very difficult to solve both numerically and analytically, since their solutions do not depend continuously on the prescribed boundary conditions. That is, a small perturbation in measured data may result in an enormous effect in the numerical solution. The Cauchy problem of the Helmholtz equations is often encountered in many branches of science and engineering $[1,19,31]$, such as wave propagation and scattering, vibration, electromagnetic field, and heat conduction.

The measured data in inverse problems of practical significance are usually observed only on a part of the boundary.

Author for correspondence: Wen Chen (e-mail: chenwen@hhu.edu.cn). * Center for Numerical Simulation Software in Engineering and Sciences, Department of Engineering Mechanics, College of Civil Engineering, Hohai University, Nanjing 210098, P.R. China

Supported by a research project funded by the National Natural Science Foundation of China (Project No. 10672051)
Therefore, the boundary-type methods have a prominent edge over the domain-type methods. And the boundary element method (BEM) with iterative or regularization techniques has been applied for the Cauchy problems in the literature [24, 25]. However, the BEM requires the evaluation of singular integrals due to the singularity of the fundamental solution, and the high-quality meshing of the irregular domain is nontrivial. Moreover, the traditional BEM requires the inner nodes in handling inhomogeneous problems and loses its essential merit to some extent for inverse problems.

To overcome mesh generation problem, facing the traditional mesh-based BEM, a variety of boundary-type meshless methods, in recent years, have been developed and attract a lot of attention, for example, method of fundamental solution (MFS) [4, 11, 22], boundary knot method (BKM) [10], plane wave method (PWM) [3, 29], collocation Trefftz method (CTM) [21] and regularized meshless method (RMM) [5, 32], among which the former three methods also do not involve the singular integration. MFS [26, 30, 33], BKM [17, 18], PWM [16] and CTM [23] have been successfully applied to the inverse Cauchy problems. All these boundary meshless methods can solve homogeneous problems with boundary-only discretization, but require inner nodes to handle inhomogeneous problems.

The dual reciprocity method (DRM) [28] and the multiple reciprocity method (MRM) [27] are two techniques of the most popular in handling inhomogeneous problems in conjunction with boundary discretization methods. The striking advantage of the MRM over the DRM is that it does not require inner nodes at all for evaluating the particular solution. To take advantage of this truly boundary-only merit, Chen [6] developed the MRM-based meshless boundary particle method (BPM). The BPM is truly meshless and integrationfree and applies either high-order nonsingular general solutions or singular fundamental solutions $[9,15]$ as the radial basis functions. Recently, Chen and Jin [7, 8] develop the recursive composite multiple reciprocity method (RC-MRM) to expand the application territory of the BPM to a broader territory of inhomogeneous problems.

In this paper, we extend the RC-MRM BPM combined with truncated singular value decomposition (TSVD) regularization techniques to inverse Cauchy problems of different in- 
homogeneous Helmholtz equations. The Generalized crossvalidation $(\mathrm{GCV})$ is one of strategies to estimate an appropriate regularization parameter of the TSVD and is employed in our numerical experiments.

The remainder of the paper is organized as follows. Section II introduces the Cauchy problem mathematically. Section III describes the boundary particle method for the Cauchy problem associated with inhomogeneous Helmholtz equations, followed by the Section IV to numerically examine the efficiency and stability of the present method in smooth and piecewise smooth boundary examples. Finally, some conclusions are given in Section V.

\section{FORMULATION OF INVERSE CAUCHY PROBLEM}

The paper is concerned with the inverse Cauchy problem, in which the unknown boundary condition on a part of boundary is to be estimated. Consider an simply-connected open bounded domain in $\Omega \subset R^{d}$, where $d$ denotes the dimensionality of the space, and assume that $\Omega$ is bounded by a smooth or piecewise smooth boundary $\partial \Omega$. Then the mathematical formulation of the Cauchy problem can be presented as

$$
\left(\Delta+k^{2}\right) u(x)=f(x), \quad x \in \Omega,
$$

where $\Delta$ denotes the Laplace operator, $k$ represents a complex number, and $f(x)$ is a known function. In this study, we consider only the cases with real or purely imaginary $k$, called the Helmholtz and modified Helmholtz equations hereafter, respectively. The Helmholtz equation arises frequently in various physical problems, such as acoustics, electromagnetics and vibration, while the modified Helmholtz equation is encountered in heat-conduction, diffusion and convection-diffusion problems.

The inverse Cauchy problem under investigation requires solving (1) subjected to the two types of boundary conditions prescribed on the accessible boundary

$$
\begin{array}{ll}
u(x)=g_{1}(x), & x \in \Gamma_{1}, \\
\frac{\partial u(x)}{\partial n}=g_{2}(x), & x \in \Gamma_{1},
\end{array}
$$

where $g_{1}$ and $g_{2}$ are the prescribed functions, $\Gamma_{1}$ denotes the non-zero measurable boundary part, and $n$ denotes the unit outward normal vector, and (2) and (3) denote the Dirichlet boundary condition and the Neumann boundary condition, respectively. A necessary condition for the above inverse Cauchy problem to be identifiable is that the known boundary part is longer than the under-specified boundary part $\Gamma_{2}$. And in this study, we focus on determining the underprescribed functions on the inaccessible boundary $\Gamma_{2}$.

\section{BOUNDARY PARTICLE METHOD BASED ON RC-MRM}

The boundary particle method (BPM) is a truly boundary-only collocation scheme, whose basis function is the high-order nonsingular general solution or singular fundamental solution. The method can be illustrated as a two-step approach. Firstly, a particular solution to the inhomogeneous problem is found, and secondly its homogeneous solution is obtained. Hence the solution to the equation can be split as two parts.

$$
u(x)=u_{p}(x)+u_{h}(x)
$$

where $u_{p}(x)$ and $u_{h}(x)$ are the particular and homogeneous solutions, respectively. To be more precise, $u_{p}(x)$ satisfies

$$
\left(\Delta+k^{2}\right) u_{p}(x)=f(x), \quad x \in \Omega,
$$

but it does not necessarily satisfy the boundary condition. And $u_{h}(x)$ satisfies the following homogeneous equations

$$
\begin{gathered}
\left(\Delta+k^{2}\right) u_{h}(x)=0, \quad x \in \Omega, \\
\mathrm{B}_{1} u_{h}(x)=g_{1}(x)-\mathrm{B}_{1} u_{p}(x), \quad x \in \Gamma_{1}, \\
\mathrm{~B}_{2} u_{h}(x)=g_{2}(x)-\mathrm{B}_{2} u_{p}(x), \quad x \in \Gamma_{1},
\end{gathered}
$$

To evaluate the particular solution, this study uses the recursive composite multiple reciprocity method (RC-MRM) to avoid the inner nodes [7, 8]. However, unlike the original MRM [27], the RC-MRM annihilates the inhomogeneous term by using a composite differential operator which can be different from the one in the original governing equation. It eliminates the inhomogeneous term $f(x)$ in (5) by iterative differentiations

$$
L_{m} \ldots L_{2} L_{1}\{f(x)\} \cong 0
$$

where $L_{1}, L_{2}, \ldots L_{m}$ are differential operators of the same or different kinds. According to (7), Eq. (5) can be transformed into the following high-order homogeneous problem:

$$
\left\{\begin{array}{lc}
L_{m} \ldots L_{2} L_{1}\left(\Delta+k^{2}\right) u(x)=0 & x \in \Omega \\
\vdots & \\
L_{2} L_{1}\left(\Delta+k^{2}\right) u(x)=L_{2} L_{1}(f(x)) & x \in \partial \Omega \\
L_{1}\left(\Delta+k^{2}\right) u(x)=L_{1}(f(x)) & x \in \partial \Omega \\
\left(\Delta+k^{2}\right) u(x)=f(x) & x \in \partial \Omega
\end{array} .\right.
$$

Now the particular solution $u_{p}(x)$ can be evaluated by the high-order homogeneous equations (8). Hence the Cauchy 
Table 1. Nonsingular general or harmonic solution of typical differential operators.

\begin{tabular}{|c|c|c|}
\hline$L$ & 2 dimension & 3 dimension \\
\hline$\Delta$ & $\exp \left(-c\left(x_{1}^{2}-x_{2}^{2}\right)\right) \cos \left(2 c x_{1} x_{2}\right)$ & $\begin{array}{l}\exp \left(-c\left(x_{1}^{2}-x_{2}^{2}\right)\right) \cos \left(2 c x_{1} x_{2}\right)+\exp \left(-c\left(x_{2}^{2}-x_{3}^{2}\right)\right) \cos \left(2 c x_{2} x_{3}\right)+ \\
\exp \left(-c\left(x_{3}^{2}-x_{1}^{2}\right)\right) \cos \left(2 c x_{1} x_{3}\right)\end{array}$ \\
\hline$\Delta+k^{2}$ & $J_{0}(k r)$ & $\frac{\sin (k r)}{r}$ \\
\hline$\Delta-k^{2}$ & $I_{0}(k r)$ & $\frac{\sinh (k r)}{r}$ \\
\hline$\Delta+\mathbf{v} \bullet \nabla-k^{2}$ & $I_{0}(k r) e^{-\frac{\mathbf{v} \cdot \mathbf{r}}{2}}$ & $\frac{e^{-\frac{\mathbf{v} \cdot r}{2}} \sinh (k r)}{r}$ \\
\hline
\end{tabular}

problem associated with inhomogeneous equation requires solving two homogeneous equations (6) and (8). The corresponding homogeneous solutions of different orders can be approximated by a linear combination of nonsingular general solutions of the governing differential operator

$$
\tilde{u}(x)=\sum_{j=1}^{N} a_{j} u^{*}\left(x-y_{j}\right), \quad x \in \Omega,
$$

where $N$ represents the number of source points, $\left\{y_{j}\right\}$ denotes the source points, $\left\{a_{j}\right\}$ are coefficients to be determined, and $u^{*}(x)$ means the nonsingular general or harmonic solution of the typical governing differential operator.

Table 1 displays nonsingular general or harmonic solutions of typical differential operators $[9,15]$. There is no suitable nonsingular general solution for Laplace equation, and this study uses the nonsingular harmonic function $[8,14]$. Here $c$ in harmonic function of Laplace operator is the shape parameter, $\nabla$ denotes the gradient operator, $\mathbf{v}$ and $\mathbf{r}$, respectively, represent the velocity vector and distance vector, and $r$ denotes the Euclidean distance. $I_{0}$ and $J_{0}$ represent the Bessel and modified Bessel functions of the first kind of order zero, respectively.

\section{NUMERICAL RESULTS AND DISCUSSIONS}

The inverse Cauchy problems are of ill-posedness in nature. Thus, the solution is unstable regarding a small perturbation on the over-specified boundary $\Gamma_{1}$. Hence the standard methods, such as the Gauss elimination method and the Leastsquares method, often fail to yield satisfactory results due to the combination of the ill-conditioning interpolation discertization matrix and data noise. A few techniques available today mitigate this effect, such as the domain decomposition method [2], preconditioning technique based on approximate cardinal basis function, the fast multiple method [19], regularization methods (e.g., the truncated singular value decomposition (TSVD)) [12]. It is noted that the TSVD with GCV function choice criterion is employed to obtain accurate and stable results. The Generalized cross-validation $(\mathrm{GCV})$ is one of strategies to estimate an appropriate regularization parameter of the TSVD.

To examine the BPM in conjunction with TSVD for the inverse Cauchy problems, this section presents numerical results of four benchmark examples of 2D inhomogeneous Helmholtz problems. All the computational codes are programmed in MATLAB, partially including the MATLAB TSVD code developed by Hansen [13] for the discrete ill-posed problem.

This study involves the two types of solution domain. The first computational domain is a smooth circular domain centered at origin $\Omega_{1}=\left\{\left(x_{1}, x_{2}\right) \mid x_{1}^{2}+x_{2}^{2}<1\right\}$ with the measured boundary part $\Gamma_{1}=\{(r, \theta) \mid r=1,0 \leq \theta \leq 3 \pi / 2\}$, and the unmeasured boundary part $\Gamma_{2}=\{(r, \theta) \mid r=1,3 \pi / 2 \leq \theta \leq 2 \pi\}$, where $(r, \theta)$ is the plane polar coordinate.

The second computational domain is a piecewise smooth square $\Omega_{2}=\left\{\left(x_{1}, x_{2}\right) \mid 0<x_{1}, x_{2}<1\right\}$ with the measured boundary part $\Gamma_{1}=\left\{\left(0, x_{2}\right) \mid 0 \leq x_{2} \leq 1\right\} \cup\left\{\left(1, x_{2}\right) \mid 0 \leq x_{2} \leq 1\right\} \cup\left\{\left(x_{1}, 1\right) \mid 0 \leq x_{1} \leq 1\right\}$ and the unmeasured boundary part $\Gamma_{2}=\left\{\left(x_{1}, 0\right) \mid 0 \leq x_{1} \leq 1\right\}$.

Example 1. Inhomogeneous Helmholtz equation on abovedefined smooth circular domain $\Omega_{1}$ with $k=\sqrt{2}$. The exact solution $u(x)=\sin \left(x_{1}\right) \sin \left(x_{2}\right)+x_{1}+\sin \left(x_{2}\right)$, and the forcing term $f(x)=2 x_{1}+\sin \left(x_{2}\right)$, and its annihilating operator of this case in $(7)$ is $(\Delta+1) \Delta$.

Example 2. Inhomogeneous Helmholtz equation on abovedefined piecewise smooth square $\Omega_{2}$, with $k=\sqrt{2}$. The exact solution $u(x)=\sin \left(x_{1}\right) \sin \left(x_{2}\right)+x_{1}+\sin \left(x_{2}\right)$, and the forcing term $f(x)=2 x_{1}+\sin \left(x_{2}\right)$, and its annihilating operator of this case in $(7)$ is $(\Delta+1) \Delta$.

Example 3. Inhomogeneous Helmholtz equation on abovedefined circular domain $\Omega_{1}$, with $k=\sqrt{2}$. The exact solution 
is $u(x)=x_{1} \sin \left(\sqrt{2} x_{2}\right)+x_{1}-x_{2}+\exp \left(x_{1}\right)$, and the forcing term $f(x)=2 x_{1}-2 x_{2}+3 \exp \left(x_{1}\right)$, and its annihilating operator of this case in (7) is $(\Delta-1) \Delta$.

Example 4. Inhomogeneous Helmholtz equation on abovedefined square $\Omega_{2}$, with $k=\sqrt{2}$. The exact solution $u(x)=$ $\sin \left(x_{1}\right) \cosh \left(\sqrt{3} x_{2}\right)+\cos \left(x_{1}\right) \sinh \left(\sqrt{3} x_{2}\right)+x_{1}^{2}-x_{2}^{3}$, and the forcing term $f(x)=2-2 x_{1}^{2}-6 x_{2}+2 x_{2}^{3}$, and its annihilating operator of this case in (7) is $\Delta^{2}$.

The measured data in practical problems always goes with errors. To investigate the stability of the BPM, the man-made noisy data is generated by

$$
\begin{aligned}
& \bar{g}_{1}(x)=g_{1}(x)(1+\operatorname{randn}(i) e), \\
& \bar{g}_{2}(x)=g_{2}(x)(1+\operatorname{randn}(i) e),
\end{aligned}
$$

where $g_{1}$ and $g_{2}$ denote the prescribed function with the exact data given in (2) and (3). The random number is chosen with a standard normal distribution, which is fixed at each example, and $e$ denotes the noise level.

The numerical accuracy is calculated by the relative root mean square errors

$$
\begin{aligned}
\operatorname{Rer}(u) & =\frac{\sqrt{\frac{1}{N T} \sum_{i=1}^{N T}\left(\tilde{u}\left(x_{i}\right)-u\left(x_{i}\right)\right)^{2}}}{\sqrt{\frac{1}{N T} \sum_{i=1}^{N T} u^{2}\left(x_{i}\right)}}, \\
\operatorname{Rerr}(q) & =\frac{\sqrt{\frac{1}{N T} \sum_{i=1}^{N T}\left(\tilde{q}\left(x_{i}\right)-q\left(x_{i}\right)\right)^{2}}}{\sqrt{\frac{1}{N T} \sum_{i=1}^{N T} q^{2}\left(x_{i}\right)}},
\end{aligned}
$$

where $u\left(x_{i}\right)\left(q\left(x_{i}\right)\right)$ and $\tilde{u}\left(x_{i}\right)\left(\tilde{q}\left(x_{i}\right)\right)$ are respectively the analytical and numerical results evaluated at $x_{i}$, flux $q\left(x_{i}\right)=$ $\frac{\partial u\left(x_{i}\right)}{\partial n}, n$ denotes the unit outward normal vector, and $N T$ is the number of test nodes on the under-specified boundary $\Gamma_{2}$. In this study, $N T$ is 40 , and the points are distributed uniformly on the under-specified boundary $\Gamma_{2}$.

Figure 1(a) shows the BPM convergence curves for example 1 . Roughly speaking, with an increasing number of nodes on the prescribed Neumann and Dirichlet boundary conditions, the numerical accuracy of estimated solution improves in an oscillatory fashion. The minimum relative root mean square errors are found less than $10^{-2}$. We can see that the estimated accuracy of $u(x)$ on the under-specified boundary is a little higher than that of flux $q(x)$ with the increasing measured points.

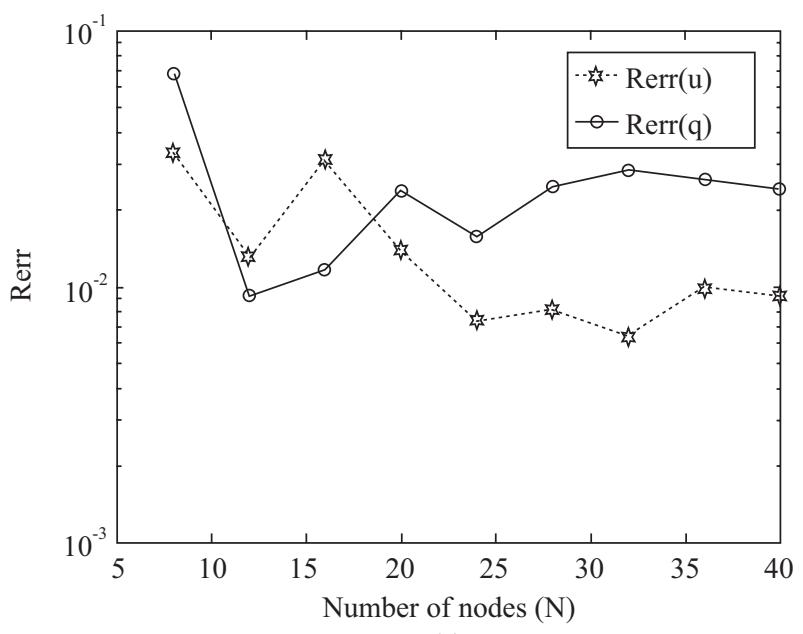

(a)

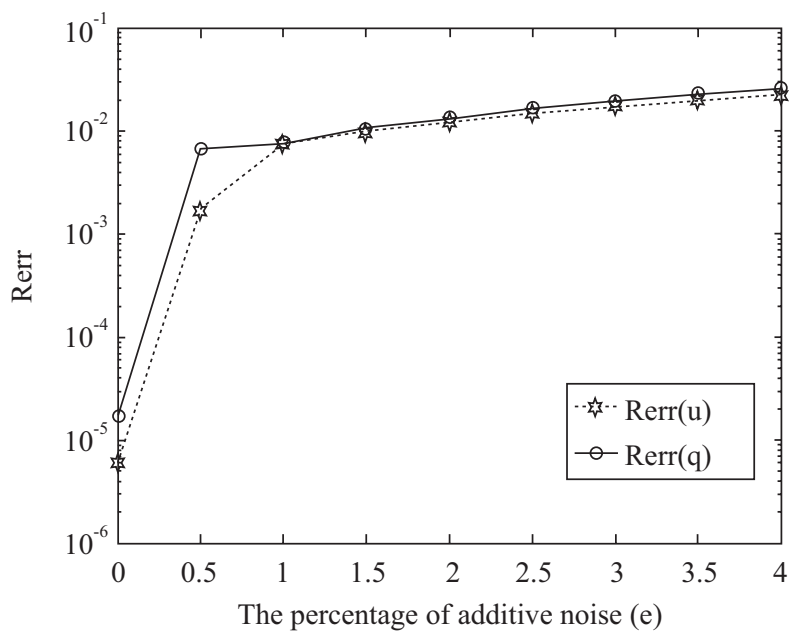

(b)

Fig. 1. BPM numerical accuracy variation with respect to (a) the number of measurement points with $2 \%$ noise level, and (b) the noise level percentage when using 36 measurement points for example 1.

Figure 1(b) depicts the numerical accuracy variation with respect to various levels of noise in the data. It is observed that the curves of the relative root mean square error decays with the decreasing noise data. It is found that the numerical solutions achieve best accuracy with noise-free data.

Figures 2-5 illustrate exact solutions and BPM estimated results under various levels of noise for examples 2-4, respectively. It can be seen from Figs. 2 and 3 that estimated results of examples 2 and 3 agree quite well with the analytical solutions under $2 \%$ noise level. It is also noted from Figs. 4 and 5 that numerical estimated results of example 4 are not satisfactory using 36 boundary nodes, but are significantly improved when the more boundary nodes (75) are used.

Table 2 compares the numerical accuracy of the BPM and the reference method [18]. It is seen that these two methods have similar solution accuracy. It is stressed that the reference method [18] requires using the points inside and outside domain to evaluate the particular solution, whereas the BPM can 


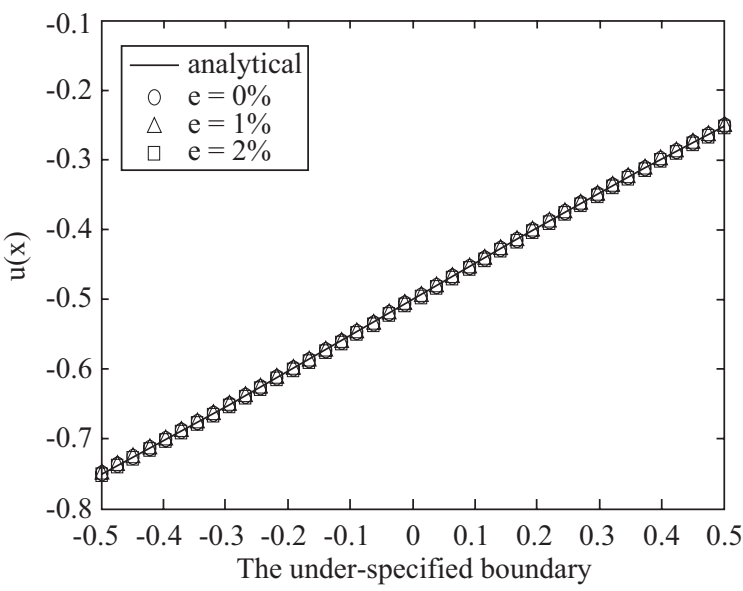

(a)

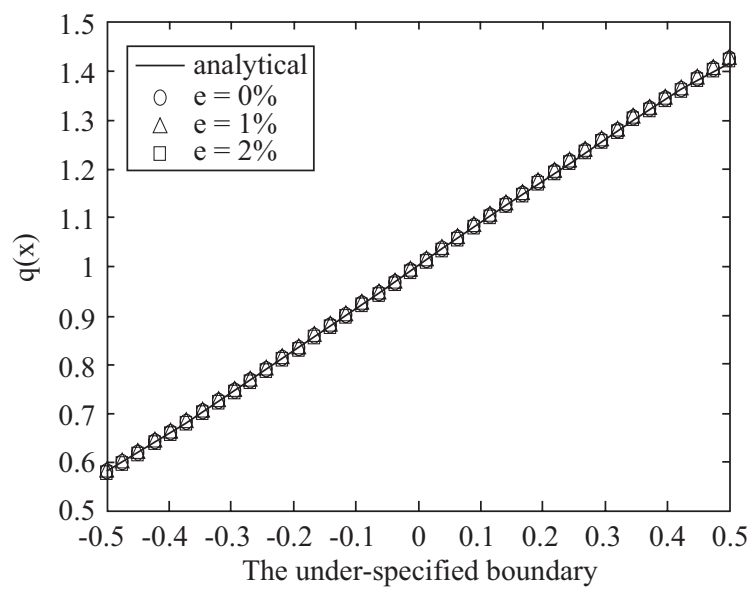

(b)

Fig. 2. (a) The analytical solution $u(x)$ and the BPM solution $\tilde{u}(x)$, and (b) the analytical solution $q(x)$ and the BPM solution $\tilde{q}(x)$ using 36 measurement points for example 2 with $0 \%, 1 \%$ and $2 \%$ noise levels.

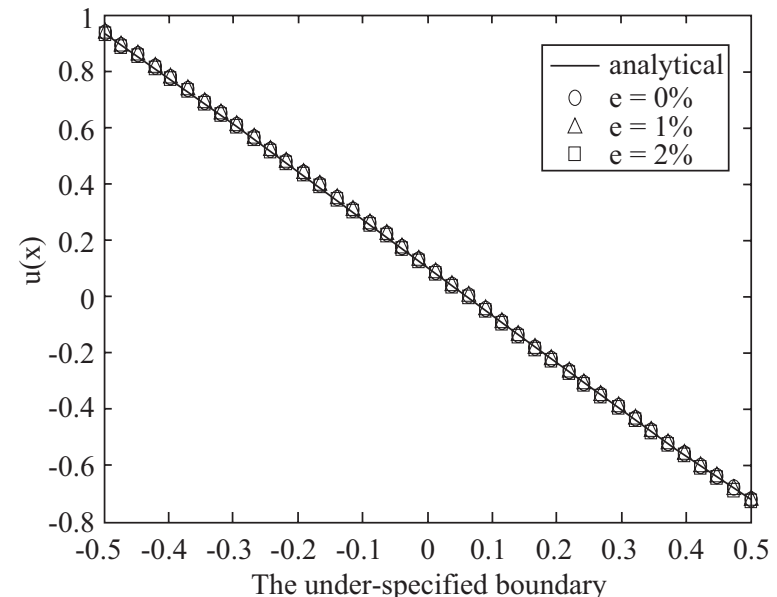

(a)

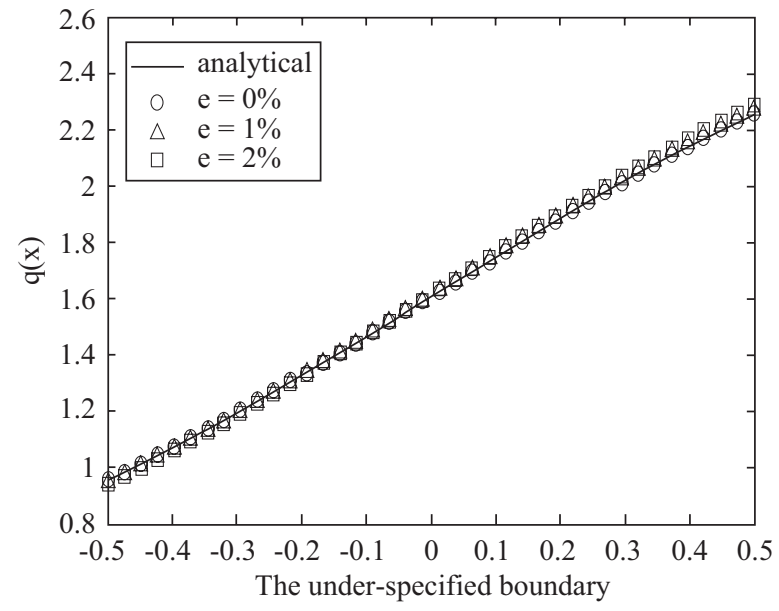

(b)

Fig. 3. (a) The analytical solution $u(x)$ and the BPM solution $\tilde{u}(x)$, and (b) the analytical solution $q(x)$ and the BPM solution $\tilde{q}(x)$ using 36 measurement points for example 3 with $0 \%, 1 \%$ and $2 \%$ noise levels.

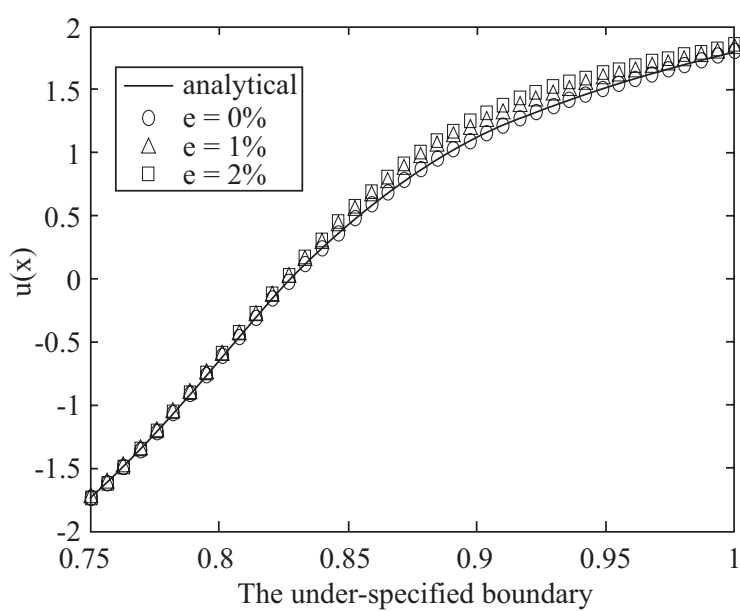

(a)

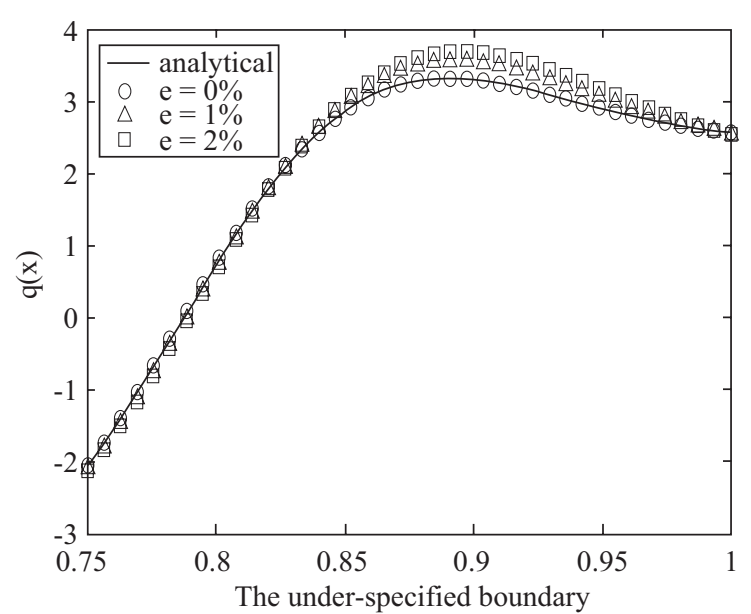

(b)

Fig. 4. (a) The analytical solution $u(x)$ and the BPM solution $\tilde{u}(x)$, and (b) the analytical solution $q(x)$ and the BPM solution $\tilde{q}(x)$ using 36 measurement points for example 4 with $0 \%, 1 \%$ and $2 \%$ noise levels. 


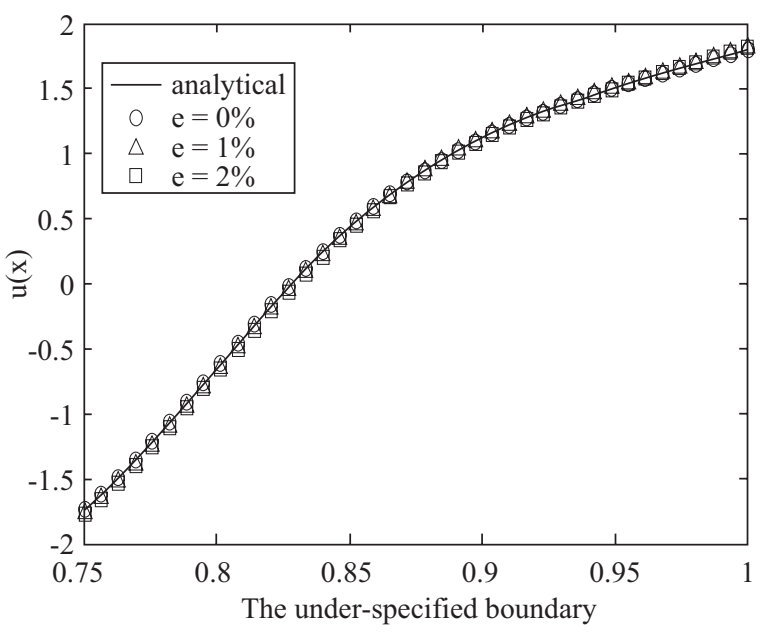

(a)

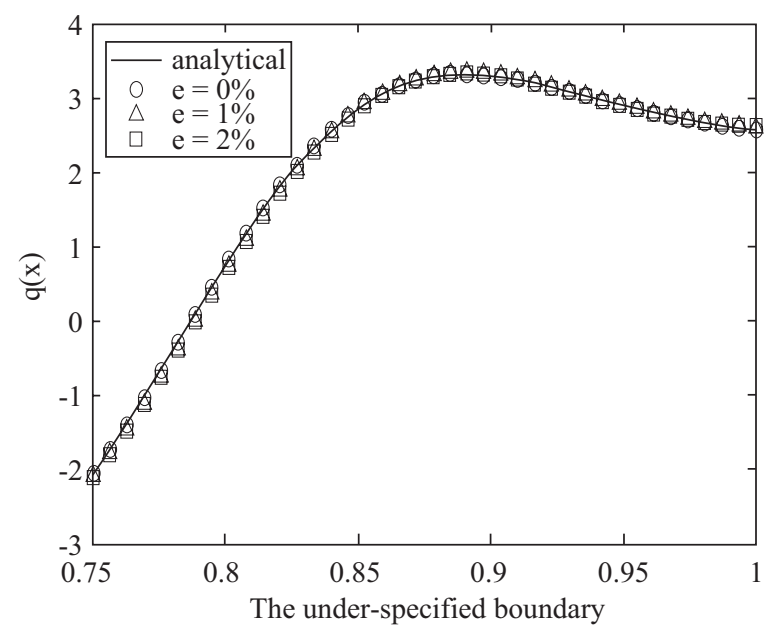

(b)

Fig 5. (a) The analytical solution $u(x)$ and the BPM solution $\tilde{u}(x)$, and (b) the analytical solution $q(x)$ and the BPM solution $\tilde{q}(x)$ using 75 measurement points for example 4 with $0 \%, 1 \%$ and $2 \%$ noise levels.

Table 2. BPM ( 36 boundary nodes) versus BKM ( 20 boundary nodes +400 additional points) solutions when $2 \%$ noise measurement data of Examples 3 and 4.

\begin{tabular}{|c|c|c|c|c|c|c|}
\hline \multirow{2}{*}{ Example } & \multicolumn{3}{|c|}{ Boundary particle method } & \multicolumn{3}{|c|}{ Boundary knot method [17] } \\
\hline & Cond & $\operatorname{Rerr}(u)$ & $\operatorname{Rerr}(q)$ & Cond & $\operatorname{Rerr}(u)$ & $\operatorname{Rerr}(q)$ \\
\hline 3 & $1.49 \mathrm{E}+17$ & $9.40 \mathrm{E}-03$ & $1.00 \mathrm{E}-02$ & $3.88 \mathrm{E}+18$ & $3.45 \mathrm{E}-02$ & $2.42 \mathrm{E}-02$ \\
\hline 4 & $3.43 \mathrm{E}+18$ & 8.23E-02 & 8.71E-02 & $3.59 \mathrm{E}+18$ & 2.94E-02 & $4.52 \mathrm{E}-02$ \\
\hline
\end{tabular}

solve inhomogeneous Cauchy problems with boundary-only discretization. It does not require any additional nodes, which is especially attractive to solve the inverse and optimization problems of high-dimensional irregular domains.

\section{CONCLUSIONS}

This paper extends the boundary particle method in conjunction with the truncated singular value decomposition regularization technique to the inverse Cauchy problem of inhomogeneous Helmholtz equations. Our numerical verification shows that the present numerical scheme can obtain an accurate and stable numerical solution and is convergent with respect to decreasing levels of noise. It is stressed that the present method is a truly boundary-only numerical scheme and solves the inverse Cauchy problems of the inhomogeneous Helmholtz equations without using any inner nodes, which is far more attractive than the other existing numerical methods in the solution of inverse and optimization problems, where only a part of boundary data are usually accessible.

\section{REFERENCES}

1. Bai, M. R., "Application of BEM-based acoustic holography to radiation analysis of sound sources with arbitrarily shaped geometries," Journal of the Acoustical Society of America, Vol. 92, pp. 533-549 (1992).
2. Beatson, R. K., Cherrie, J. B., and Mouat, C. T., "Fast fitting of radial basis functions: methods based on preconditioned GMRES iteration," Advances in Computational Mathematics, Vol. 11, pp. 253-270 (1999).

3. Bogaert, I., Pissoort, D., and Olyslager, F., "A normalized plane wave method for 2d Helmholtz problems," Microwave and Optical Technology Letters, Vol. 48, No. 2, pp. 237-243 (2006).

4. Chen, C. S., Cho, H. A., and Golberg, M. A., "Some comments on the ill-conditioning of the method of fundamental solutions," Engineering Analysis with Boundary Elements, Vol. 30, pp. $405-410$ (2006).

5. Chen, K. H., Chen, J. T., and Kao, J. H., "Regularized meshless method for antiplane shear problems with multiple inclusions," International Journal for Numerical Methods in Engineering, Vol. 73, pp. 1251-1273 (2007).

6. Chen, W., "Meshfree boundary particle method applied to Helmholtz problems," Engineering Analysis with Boundary Elements, Vol. 26, pp. 577-581 (2002).

7. Chen, W., "Distance function wavelets - Part III: "Exotic" transforms and series. Research report of Simula Research Laboratory," CoRR preprint, http://arxiv.org/abs/cs.CE/0206016, 2002.

8. Chen, W. and Jin, B. T., "A truly boundary-only meshfree method for inhomogeneous problems," Proceeding of The 2nd ICCES Special Symposium on Meshless Methods (2006).

9. Chen, W., Shen, Z. J., and Yuan, G. W., "General solutions and fundamental solutions of varied orders to the vibrational thin, the Berger, and the Winkler plates," Engineering Analysis with Boundary Elements, Vol. 29, pp. 699-702 (2005).

10. Chen, W. and Tanaka, M., "A meshless, exponential convergence, integration-free, and boundary-only RBF technique," Computers and Mathematics with Applications, Vol. 43, pp. 379-391 (2002).

11. Fairweather, G. and Karageorghis, A., "The method of fundamental solutions for elliptic boundary value problems," Advances in Computational Mathematics, Vol. 9, pp. 69-95 (1998). 
12. Hansen, P. C., "The truncated SVD as a method for regularization," BIT Numerical Mathematics, Vol. 27, No. 4, pp. 534-553 (1987).

13. Hansen, P. C., "Regularization tools: a Matlab package for analysis and solution of discrete ill-posed problems," Numerical Algorithms, Vol. 6, pp. 1-35 (1994).

14. Hon, Y. C. and Wu, Z., "A numerical computation for inverse determination problem," Engineering Analysis with Boundary Elements, Vol. 24, pp. 599-606 (2000).

15. Itagaki, M., "Higher order three-dimensional fundamental solutions to the Helmholtz and the modified Helmholtz equations," Engineering Analysis with Boundary Elements, Vol. 15, pp. 289-293 (1995).

16. Jin, B. T. and Marin, L., "The plane wave method for inverse problems associated with Helmholtz-type equations," Engineering Analysis with Boundary Elements, Vol. 32, No. 3, pp. 223-240 (2008).

17. Jin, B. T. and Zheng, Y., "Boundary knot method for some inverse problems associated with Helmholtz equation," International Journal for Numerical Methods in Engineering, Vol. 62, No. 12, pp. 1636-1651 (2005).

18. Jin, B. T. and Zheng, Y., "Boundary knot method for the Cauchy problem associated with the inhomogeneous Helmholtz equation," Engineering Analysis with Boundary Elements, Vol. 29, No. 10, pp. 925-935 (2005).

19. Kim, B. K. and Ih, J. G., "On the reconstruction of the vibro-acoustic field over the surface enclosing an interior space using the boundary element method," Journal of the Acoustical Society of America, Vol. 100, pp. 3003-3016 (1996).

20. Ling, L. and Kansa, E. J., "Preconditioning for radial basis functionswith domain decomposition methods," International Workshop on Meshfree Methods, Portugual, Instituto Superior Technico Lisbon, 2003.

21. Liu, C. S., "A modified Trefftz method for two-dimensional Laplace equation considering the domain's characteristic length," Computer Modeling in Engineering \& Sciences, Vol. 21, pp. 53-65 (2007).

22. Liu, C. S., "Improving the ill-conditioning of the method of fundamental solutions for 2D Laplace equation," Computer Modeling in Engineering \& Sciences, Vol. 28, pp. 77-93 (2008).

23. Liu, C. S., "A modified collocation Trefftz method for the inverse Cauchy problem of Laplace equation," Engineering Analysis with Boundary Elements, Vol. 32, pp. 778-785 (2008).
24. Marin, L., Elliott, L., Heggs, P. J., Ingham, D. B., Lesnic, D., and Wen, $\mathrm{X} .$, "An alternating iterative algorithm for the Cauchy problem associated to the Helmholtz equation," Computer Methods in Applied Mechanics and Engineering, Vol. 192, pp. 709-722 (2003).

25. Marin, L., Elliott, L., Heggs, P. J., Ingham, D. B., Lesnic, D., and Wen, $\mathrm{X}$., "Comparison of regularization methods for solving the Cauchy problem associated with the Helmholtz equation," International Journal for Numerical Methods in Engineering, Vol. 60, No. 11, pp. 1933-1947 (2004).

26. Marin, L. and Lesnic, D., "The method of fundamental solutions for the Cauchy problem associated with two-dimensional Helmholtz-type equations," Computers \& Structures, Vol. 83, pp. 267-278 (2005).

27. Nowak, A. J. and Partridge, P. W., "Comparison of the dual reciprocity and the multiple reciprocity methods," Engineering Analysis with Boundary Elements, Vol. 10, pp. 155-160 (1992).

28. Patridge, P. W., Brebbia, C. A., and Wrobel, L. W., The Dual Reciprocity Boundary Element Method, Computational Mechanics Publication, Southampton, 1992.

29. Pinkus, A., "Approximating by ridge functions," In: Le Mehaute, A., Rabut, C., and Schumaker, L. L., (eds.), Surface Fittin and Multiresolution Methods, Nashville, TN: Vanderbilt University Press, pp. 279-292 (1997).

30. Wei, T., Hon, Y. C., and Ling, L., "Method of fundamental solutions with regularization techniques for Cauchy problems of elliptic operators," Engineering Analysis with Boundary Elements, Vol. 31, No. 4, pp. 373-385 (2007).

31. Wu, S. R. and Yu, J., "Application of BEM-based acoustic holography to radiation analysis of sound sources with arbitrarily shaped geometries," Journal of the Acoustical Society of America, Vol. 104, pp. 2054-2060 (1998).

32. Young, D. L., Chen, K. H., and Lee, C. W., "Novel meshfree method for solving the potential problems with arbitrary domain," Journal of Computational Physics, Vol. 209, pp. 290-321 (2005).

33. Young, D. L., Tsai, C. C., Fan, C. M., and Chen, C. W., "The method of fundamental solutions and condition number analysis for inverse problem of Laplace equation," Computers and Mathematics with Applications, Vol. 55, pp. 1189-1200 (2008). 